\title{
A Ratio-dependent Eco-epidemiological Model Incorporating a Prey Refuge
}

\author{
A. K. PAL ${ }^{1, *}$, G. P. Samanta ${ }^{2}$ \\ ${ }^{1}$ Department of Mathematics, S. A. Jaipuria College, Kolkata-700005, India \\ ${ }^{2}$ Department of Mathematics, Bengal Engineering and Science University, Shibpur, Howrah - 711 103, India \\ *Corresponding Author: akpal_2002@yahoo.co.in
}

Copyright (C)2013 Horizon Research Publishing All rights reserved.

\begin{abstract}
The present paper deals with the problem of a ratio-dependent predator-prey model incorporating a prey refuge with disease in the prey-population. We assume the predator population will prefer only infected population for their diet as those are more vulnerable. Dynamical behaviours such as boundedness, local and global stability are addressed. We have also studied the effect of discrete time delay on the model. Computer simulations are carried out to illustrate our analytical findings.
\end{abstract}

Keywords Eco-epidemiological Model, Ratio-dependent, Refuge, Local Stability, Global Stability, Hopf Bifurcation

\section{Introduction}

The dynamic relationship between predator and their prey has long been and will continue to be one of the dominant topics in both ecology and mathematical ecology due to its universal existence and importance. These problems may appear to be simple mathematically at first sight, they are, in fact, often very challenging and complicated.

The formation of classical Lotka-Volterra [25,29] model was a milestone progress in the study of predator-prey interactions. Similarly, after the pioneering work of Kermack-Mckendric [20], epidemiological studies also received special attention to the researchers. Disease in ecological systems is an important issue. Anderson and May [1] were the pioneers for formulating the mathematical model on this topic. A lot of research articles have already been appeared on this subject [1,2]. Most of the studies mainly focused on parasite infection in prey population only $[5,11,14,32]$. The dynamics of predator-prey system with infection in prey population is an important study from modelling point of view. Most of predators preferentially consume diseased prey [17]. Predators harm parasite directly by consuming infected prey and also harms parasites indirectly by reducing the density of susceptible hosts. Predators behaviour can magnify this direct effect because predators often prefer infected prey over uninfected prey. So that infectious disease can be a factor to regulate human and animal population size.

It is traditionally postulated that the spread of an infectious disease in a population occurs according to the principle of mass action. For this reason, models of infectious disease with nonlinear incidence rates have been attracting considerable attention over the last two decades. The most common in the literature nonlinear incidence rate takes the form $\beta I S$, if $S(t)$ and $I(t)$ are the fractions of susceptible and infective individuals in a population. Models with this incidence rate have been studied by Liu et al. [23,24], Hethcote et al. [13] and many others. Unfortunately, there is an assumption which is often misinterpreted and misunderstood. Namely, $\beta$, it is the maximum number of infections an infective prey population can cause in a unit of time. This shows that the infection term $\beta I S$ should be replaced by $\frac{\beta I S}{S+I}$. When the total population is constant, a fact if the disease is not fatal and the model does not address vital dynamics (the normal birth and death dynamics), then the infection term $\beta I S$ may be justified but the meaning of $\beta$ becomes the encounter infection rate. Such a mechanism is better described by $\frac{\beta I S}{S+I}$ than $\beta I S$ (Hwang and Kuang [18]).

To study the predator-prey system, the most crucial element is the choice of "functional response" or "trophic function", the function that describes the number of prey consumed per predator per unit time for given quantities 
of prey and predator. Various forms of functional responses has becomes the focus of considerable attention from time to time in ecological literature. Much early works were concerned with the way in which this function varies with prey density (e.g., the so called Lotka-Volterra [25,29] and Holling Type-II [15] functional responses). A predator-dependent functional response, which is a function of the ratio of the prey and predator, is known as a ratio-dependent functional response. Predator-prey models with such ratio-dependent functional response are strongly supported by numerous field and laboratory experiments [3,7,28,31].

The research of the hiding behaviour of preys have been incorporated as a new ingredient of prey-predator models and its consequences on the dynamics of prey-predator interactions can be recognized as one of the major issues in both applied mathematics and theoretical ecology. In nature, prey populations often access to areas where they are safe from their predators. Such refugia are usually playing two significant role, serving both to reduce the chance of extinction due to predation and to damp prey-predator oscillations. These are therefore a potentially important means of increasing species richness in natural communities and of stabilizing population sizes, biomass and productivity. It is well known that many more attentions have paid on the effects of a prey refuge for predator-prey system. Predator-prey interactions often exhibit spatial refuge which afford the prey some degree of protection from predation and reduce the chance of extinction due to predation $[16,19,26,30]$. Hassel [12] showed that adding a large refuge to a model, which exhibited divergent oscillations in the absence of refuge, replaced the oscillatory behaviour with a stable equilibrium. These mathematical models and a number of experiments indicate that refuge have a stabilizing effect on predator-prey interactions.

Time delays of one type or another have been incorporated into epidemiological models by many authors $[8,10,21,27]$. In general, delay-differential equations exhibit much more complicated dynamics than ordinary differential equations since a time-delay could cause a stable equilibrium to become unstable and cause the populations to fluctuate.

In this paper, we have investigated the dynamical behaviour of a ratio-dependent predator-prey systems with infection in prey population, and the effect of refuge in the infected prey. Here we have studied the boundedness, local and global stabilities of the non-equilibrium points of this system. We have also considered a discrete timedelay in the interaction term of the predator equation.

The rest of the paper is structured as follows: In section 2, we present a brief sketch of the construction of the model, which may indicate the epidemiological relevance of it.In section 3, boundedness of the basic deterministic model (2.2) is discussed. Section 4 deals with the boundary equilibrium points and their stability. In section 5 , we find the necessary and sufficient condition for the existence of the interior equilibrium point $E^{*}\left(s^{*}, i^{*}, y^{*}\right)$ and study its local and global stability. Computer simulations of some solutions of the system (2.2) are also presented in this section. The occurrence of Hopf bifurcation is shown in section 6 . The effect of discrete time-delay on the system (2.2) is studied in section 7. In section 8, computer simulation of variety of numerical solutions of the system with delay is presented. Section 9 contains the general discussions of the paper.

\section{The Basic Mathematical Model}

Before we introduce the model, we should like to present the brief sketch of the construction of the model which may indicate biological relevance of it.

1. We have two populations. Let $\mathrm{N}$ denotes the population density of the prey and $\mathrm{P}$ denotes the population density of the predator, respectively, in time $\mathrm{T}$.

2. We assume that in the absence of infection, the prey population density grows according to a logistic curve with carrying capacity $K(K>0)$, with an intrinsic growth rate $R(R>0)$.

$$
\frac{d N}{d T}=R N\left(1-\frac{N}{K}\right)
$$

3. When the prey population is infected, then we assume that the total prey population $N$ divided into two classes: one is the class of susceptible preys, denoted by $S$, and the other is the class of infected preys, denoted by $I$. Therefore at any time $T$, the total prey population is $N(T)=S(T)+I(T)$.

4. We assume that the disease is spread among the prey population only and the disease is not genetically inherited and the infected populations do not recover or become immune. We assume that the infection mechanism 
follows the response function

$$
\phi(S, I)=\frac{A S I}{S+I}
$$

with $A$ as the transmission rate.

5. The infected prey $I(T)$ is removed by death (say, its death rate is positive constant $D_{1}$ )or by predation before having the possibility of reproducing. However, the infected prey population $I(T)$ still contribute with $S(T)$ towards the carrying capacity of the system.

6. The infected prey is more vulnerable than susceptible prey. We assume that the predator population consumes only infected prey with ratio-dependent Michaelis-Menten functional response function

$$
\eta(I, Y)=\frac{I Y}{\mu Y+I} \quad(\mu>0) .
$$

It is assumed that the predator has the death rate constant $D_{2}\left(D_{2}>0\right)$, and the predation coefficient $C(C>0)$. The coefficient in conversing prey into predator is $e$.

7. To protect the prey populations, we construct our model by incorporating a refuge protecting $m I$ of the infected prey, where $m \in[0,1)$ is constant. This leaves $(1-m) I$ of the infected prey available to the predator.

The above considerations motivate us to introduce an eco-epidemiological model under the framework of the following set of nonlinear ordinary differential equations :

$$
\begin{aligned}
& \frac{d S}{d T}=R S\left(1-\frac{S+I}{K}\right)-\frac{A S I}{S+I} \\
& \frac{d I}{d T}=\frac{B S I}{S+I}-D_{1} I-\frac{C(1-m) I Y}{\mu Y+(1-m) I} \\
& \frac{d Y}{d T}=-D_{2} Y+\frac{e(1-m) I Y}{\mu Y+(1-m) I}
\end{aligned}
$$

with initial data $S(0) \geq 0, \quad I(0) \geq 0, \quad Y(0) \geq 0$.

The model we have just specified has ten parameters, which makes the analysis difficult. To reduce the number of parameters and to determine which combinations of parameters control the behaviour of the system, we nondimensionalize system (2.1). We choose

$$
s=\frac{S}{K}, \quad i=\frac{I}{K}, \quad y=\frac{\mu Y}{K}, \text { and } t=R T
$$

Then system (2.1) takes the form (after some simplification)

$$
\begin{aligned}
& \frac{d s}{d t}=s\{1-(s+i)\}-\frac{a s i}{s+i}, \quad s(0) \geq 0 \\
& \frac{d i}{d t}=\frac{b s i}{s+i}-d_{1} i-\frac{c(1-m) i y}{y+(1-m) i}, \quad i(0) \geq 0 \\
& \frac{d y}{d t}=-d_{2} y+\frac{l(1-m) i y}{y+(1-m) i}, \quad y(0) \geq 0
\end{aligned}
$$

where $a=\frac{A}{R}, \quad b=\frac{B}{R}, \quad c=\frac{C}{\mu R}, \quad d_{1}=\frac{D_{1}}{R}, \quad d_{2}=\frac{D_{2}}{R}, \quad l=\frac{e}{R}$

\section{Boundedness}

In theoretical eco-epidemiology, boundedness of a system implies that a system is biologically well-behaved. The following theorem ensures the boundedness of the system (2.2):

Theorem 3.1. All solutions of system (2.2) that start in $\Re_{+}^{3}$ are uniformly bounded. 
Proof : Let, $(s(t), i(t), y(t))$ be any solution of the system (2.2).

Since,

$$
\frac{d s}{d t} \leq s(1-s)
$$

We have,

$$
\limsup _{t \rightarrow \infty} s(t) \leq 1
$$

Let,

$$
W=\frac{b}{a} s+i+\frac{c}{l} y
$$

Then,

$$
\begin{aligned}
\frac{d W}{d t} & =\frac{b}{a} s(1-s-i)-d_{1} i-\frac{c d_{2}}{l} y \\
& \leq \frac{b}{a} s-d_{1} i-\frac{c d_{2}}{l} y \\
& \leq \frac{2 b}{a}-\delta W, \quad \text { where } \delta=\min \left\{1, d_{1}, d_{2}\right\}
\end{aligned}
$$

Therefore

$$
\frac{d W}{d t}+\delta W \leq \frac{2 b}{a}
$$

Applying a theorem on differential inequalities, we obtain

$$
0 \leq W(s, i, y) \leq \frac{2 b}{a \delta}+\frac{W(s(0), i(0), y(0))}{e^{\delta t}}
$$

and for $t \rightarrow \infty$,

$$
0 \leq W \leq \frac{2 b}{a \delta}
$$

Thus, all the solutions of (2.2) enter into the region

$$
\Omega=\left\{(s, i, y): 0 \leq W \leq \frac{2 b}{a \delta}+\epsilon, \text { for any } \epsilon>0\right\} .
$$

Hence the theorem.

\section{Boundary Equilibria and their Stability}

In this section, we study the stability of the boundary equilibrium points of the system (2.2). In the following lemma we have mentioned the boundary equilibria of the system (2.2) and the condition of their existence.

Lemma 4.1. System (2.2) always has two boundary equilibrium points, namely the trivial equilibrium $E_{0}(0,0,0)$ and the axial equilibrium $E_{1}(1,0,0)$. The predator-free equilibrium point $E_{2}(\hat{s}, \hat{i}, 0)$ exists if and only if a $>1$ and $d_{1}<b<\frac{a}{a-1} d_{1}$. When this condition is satisfied, $\hat{s}, \hat{i}$ are given by

$$
\hat{s}=\frac{d_{1}}{b^{2}}\left\{b-a\left(b-d_{1}\right)\right\}, \quad \hat{i}=\frac{\left(b-d_{1}\right)}{b^{2}}\{b-a(b-d)\}
$$

In terms of original parameters of the system, the condition $a>1$ becomes $A>R$ and $d_{1}<b<\frac{a}{a-1} d_{1}$ becomes $D_{1}<B<\frac{A}{A-R} D_{1}$. This implies that if the intrinsic growth rate is less than the transmission rate and the death rate of the infected prey is less than the transmission rate $B$, then the predator becomes extinct and conversely.

Remark 4.1 : When $a=b$, the necessary and sufficient condition for the existence of the predator-free equilibrium is that the force of infection lies in the interval $\left(d_{1}, 1+d_{1}\right)$.

The system $(2.2)$ cannot be linearized at $E_{0}(0,0,0)$ and $E_{1}(1,0,0)$ and therefore local stability of $E_{0}$ and $E_{1}$ cannot be studied [8]. Therefore, we are only interested in the stability in the predator-free equilibrium point $E_{2}(\hat{s}, \hat{i}, 0)$. 
The variational matrix $V\left(E_{2}\right)$ at the equilibrium point $E_{2}$ is given by

$$
V\left(E_{2}\right)=\left[\begin{array}{ccc}
-\hat{s}+\frac{a \hat{s} \hat{i}}{(\hat{s}+\hat{i})^{2}} & -\hat{s}-\frac{a \hat{s}^{2}}{(\hat{s}+\hat{i})^{2}} & 0 \\
\frac{b \hat{i}^{2}}{(\hat{s}+\hat{i})^{2}} & -\frac{b \hat{s} \hat{i}}{(\hat{s}+\hat{i})^{2}} & -c \\
0 & 0 & l-d_{2}
\end{array}\right]
$$

The characteristic equation of $V\left(E_{2}\right)$ is

$$
\left(\lambda^{2}+M \lambda+N\right)\left(\lambda-l+d_{2}\right)=0
$$

where

$$
M=\hat{s}+\frac{(b-a) \hat{s} \hat{i}}{(\hat{s}+\hat{i})^{2}} \text { and } N=\frac{b \hat{s} \hat{i}}{\hat{s}+\hat{i}}>0
$$

The eigen values are

$$
\lambda_{1,2}=\frac{-M \pm \sqrt{M^{2}-4 N}}{2} \text { and } \lambda_{3}=l-d_{2}
$$

This implies that $E_{2}$ is locally asymptotically stable in the si-plane if and only if $M>0$. Now $E_{2}$ is asymptotically stable or unstable in the y-direction according as $l<$ or $>d_{2}$.

Remark 4.2 : When $a=b$, the above result indicate that if the predator mortality is so high that the conversion factor can't control or manage it, then the system stabilizes to the predator-free equilibrium. This is quiet natural from practical point of view.

\section{The Interior Equilibrium Point : Its Existence and Stability}

First we consider the existence and uniqueness of the interior equilibrium point $E^{*}\left(s^{*}, i^{*}, y^{*}\right)$.

Lemma 5.1. The interior equilibrium point $E^{*}\left(s^{*}, i^{*}, y^{*}\right)$ of the system (2.2) exists if and only if the following two conditions are satisfied:

$$
\text { (i) } A<l b<\frac{a A}{a-1} \text { and (ii) } l>d_{2}
$$

where $A=d_{1} l+c(1-m)\left(l-d_{2}\right)$. Furthermore, $s^{*}, i^{*}, y^{*}$ are given by

$$
s^{*}=\frac{A}{l^{2} b^{2}}\{l b-a(l b-A)\}, \quad i^{*}=\frac{(l b-A)}{l^{2} b^{2}}\{l b-a(l b-A)\}, \quad y^{*}=\frac{\left(A-l d_{1}\right)(l b-A)}{c d_{2} l^{2} b^{2}}\{l b-a(l b-A)\}
$$

From Lemma 5.1, we can observe that the interior equilibrium point $E^{*}\left(s^{*}, i^{*}, y^{*}\right)$ exists if and only if both the conditions (i) and (ii) are satisfied. If any one of the condition is violated then $E^{*}\left(s^{*}, i^{*}, y^{*}\right)$ does not exists. Now from condition $(i)$ of the lemma 5.1, we have,

$$
\begin{gathered}
A<l b<\frac{a A}{a-1} \\
\Rightarrow 1-\frac{a l\left(b-d_{1}\right)-b l}{c\left(l-d_{2}\right)}>m>1-\frac{l\left(b-d_{1}\right)}{c\left(l-d_{2}\right)}
\end{gathered}
$$

Hence, to exists the interior equilibrium point $E^{*}\left(s^{*}, i^{*}, y^{*}\right)$ the refuge constant $m$ should lies in the interval

$$
1-\frac{l\left(b-d_{1}\right)}{c\left(l-d_{2}\right)}<m<1-\frac{a l\left(b-d_{1}\right)-b l}{c\left(l-d_{2}\right)}
$$

Remark 5.1: It is to be noted that the existence of $E^{*}$ destabilizes $E_{2}$. 
The variational matrix of $(2.2)$ at $E^{*}$ is given by

$$
V\left(E^{*}\right)=\left[\begin{array}{ccc}
v_{11} & v_{12} & 0 \\
v_{21} & v_{22} & v_{23} \\
0 & v_{32} & v_{33}
\end{array}\right]
$$

where

$$
\begin{gathered}
v_{11}=-s^{*}+\frac{a s^{*} i^{*}}{\left(s^{*}+i^{*}\right)^{2}}, \quad v_{12}=-s^{*}-\frac{a s^{*^{2}}}{\left(s^{*}+i^{*}\right)^{2}}, \quad v_{21}=\frac{b i^{*^{2}}}{\left(s^{*}+i^{*}\right)^{2}}, \quad v_{22}=-\frac{b s^{*} i^{*}}{\left(s^{*}+i^{*}\right)^{2}}+\frac{c(1-m)^{2} i^{*} y^{*}}{\left\{y^{*}+(1-m) i^{*}\right\}^{2}}, \\
v_{23}=-\frac{c(1-m)^{2} i^{*^{2}}}{\left\{y^{*}+(1-m) i^{*}\right\}^{2}}, \quad v_{32}=\frac{l(1-m) y^{*^{2}}}{\left\{y^{*}+(1-m) i^{*}\right\}^{2}}, \quad v_{33}=-\frac{l(1-m) i^{*} y^{*}}{\left\{y^{*}+(1-m) i^{*}\right\}^{2}}
\end{gathered}
$$

The characteristic equation is

$$
\lambda^{3}+A_{1} \lambda^{2}+A_{2} \lambda+A_{3}=0
$$

where

$$
\begin{aligned}
& A_{1}=-\operatorname{tr}\left[V\left(E^{*}\right)\right]=-v_{11}-v_{22}-v_{33}=s^{*}+\frac{(b-a) s^{*} i^{*}}{\left(s^{*}+i^{*}\right)^{2}}+\frac{(1-m)(l-c) i^{*} y^{*}}{\left\{y^{*}+(1-m) i^{*}\right\}^{2}} \\
& A_{2}=v_{11} v_{22}+v_{11} v_{33}+v_{22} v_{33}-v_{23} v_{32}-v_{12} v_{21} \\
& A_{3}=-\operatorname{det}\left[V\left(E^{*}\right)\right]=v_{11} v_{23} v_{32}+v_{12} v_{21} v_{33}-v_{11} v_{22} v_{33}=\frac{l b(1-m)\left(1+s^{*}\right) s^{*} y^{*} i^{*^{3}}}{\left(s^{*}+i^{*}\right)^{2}\left\{y^{*}+(1-m) i^{*}\right\}^{2}}>0
\end{aligned}
$$

Now

$$
\begin{aligned}
\Delta= & A_{1} A_{2}-A_{3} \\
= & -\left(v_{11}\right)^{2} v_{22}-\left(v_{11}\right)^{2} v_{33}+v_{11} v_{12} v_{21}-\left(v_{22}\right)^{2} v_{33}-v_{11}\left(v_{22}\right)^{2}-2 v_{11} v_{22} v_{33} \\
& +v_{22} v_{12} v_{21}+v_{23} v_{32} v_{22}-v_{22}\left(v_{33}\right)^{2}-v_{11}\left(v_{33}\right)^{2}+v_{23} v_{32} v_{33}
\end{aligned}
$$

By the Routh-Hurwitz criterion, it follows that all eigenvalues of characteristic equation have negative real part if and only if

$$
A_{1}>0, \quad A_{3}>0, \Delta>0
$$

Theorem 5.1. $E^{*}$ is locally asymptotically stable if and only if $A_{1}>0$ and $\Delta>0$.

\subsection{Global Stability Analysis of $E^{*}$}

Now, we shall study the global dynamics of the system (2.2) around the positive equilibrium $E^{*}\left(s^{*}, i^{*}, y^{*}\right)$. We use Liapunov function to prove the global result.

Theorem 5.2. If $a(A-l b)+l b>0$ and $\frac{b s^{*}}{(s+i)\left(s^{*}+i^{*}\right)}-\frac{c(1-m)^{2} y^{*}}{\{y+(1-m) i\}\left\{y^{*}+(1-m) i^{*}\right\}}>0$, for all $(s, i, y) \in \Re \Re_{+}^{3}$, then $E^{*}$ is globally asymptotically stable.

Proof : Let us consider the following positive definite function about $E^{*}$ :

$$
L(s, i, y)=M\left(s-s^{*}-s^{*} \ln \frac{s}{s^{*}}\right)+\left(i-i^{*}-i^{*} \ln \frac{i}{i^{*}}\right)+N\left(y-y^{*}-y^{*} \ln \frac{y}{y^{*}}\right)
$$

where $M, N$ are positive constant to be specified later on.

Differentiating $L$ with respect to $t$ along the solution of (2.2), a little algebraic manipulation yields

$$
\begin{aligned}
\frac{d L}{d t}= & -M\left\{1-\frac{a i^{*}}{(s+i)\left(s^{*}+i^{*}\right)}\right\}\left(s-s^{*}\right)^{2}-M\left(s-s^{*}\right)\left(i-i^{*}\right) \\
& +\frac{\left(b i^{*}-A a s^{*}\right)}{(s+i)\left(s^{*}+i^{*}\right)}\left(s-s^{*}\right)\left(i-i^{*}\right)-\left\{\frac{b s^{*}}{(s+i)\left(s^{*}+i^{*}\right)}-\frac{c(1-m)^{2} y^{*}}{\{y+(1-m) i\}\left\{y^{*}+(1-m) i^{*}\right\}}\right\}\left(i-i^{*}\right)^{2} \\
& -\frac{B l(1-m) i^{*}}{\{y+(1-m) i\}\left\{y^{*}+(1-m) i^{*}\right\}}\left(y-y^{*}\right)^{2}+(1-m) \frac{B l y^{*}-c(1-m) i^{*}}{\{y+(1-m) i\}\left\{y^{*}+(1-m) i^{*}\right\}}\left(i-i^{*}\right)\left(y-y^{*}\right)
\end{aligned}
$$


We now choose

$$
M=\frac{b i^{*}}{a s^{*}} \text { and } N=\frac{c(1-m) i^{*}}{l y^{*}}
$$

Then

$$
\begin{aligned}
\frac{d L}{d t}= & -\frac{b i^{*}}{a s^{*}}\left\{1-\frac{a i^{*}}{(s+i)\left(s^{*}+i^{*}\right)}\right\}\left(s-s^{*}\right)^{2}-\frac{c(1-m)^{2} i^{*^{2}}}{y^{*}\{y+(1-m) i\}\left\{y^{*}+(1-m) i^{*}\right\}}\left(y-y^{*}\right)^{2} \\
& -\frac{b i^{*}}{a s^{*}}\left(s-s^{*}\right)\left(i-i^{*}\right)-\left\{\frac{b s^{*}}{(s+i)\left(s^{*}+i^{*}\right)}-\frac{c(1-m)^{2} y^{*}}{\{y+(1-m) i\}\left\{y^{*}+(1-m) i^{*}\right\}}\right\}\left(i-i^{*}\right)^{2}
\end{aligned}
$$

Now the condition $a(A-l b)+l b>0$ implies that

$$
1-\frac{a i^{*}}{(s+i)\left(s^{*}+i^{*}\right)}>0
$$

Therefore $\frac{d L}{d t}$ is negative definite and consequently $L$ is a Lyapunov function with respect to all solutions in $\Re_{+}^{3}$. Hence the theorem.

\section{Model with Discrete Delay}

It is already mentioned that time-delay is an important factor in biological system. It is also reasonable to assume that the effect of the infected prey on the predator population will not be instantaneous, but mediated by some discrete time lag $\tau$ required for incubation. As a starting point of this section, we consider the following generalization of the model (2.2) involving discrete time-delay :

$$
\begin{aligned}
& \frac{d s}{d t}=s\{1-(s+i)\}-\frac{a s i}{s+i}, \quad s(0) \geq 0 \\
& \frac{d i}{d t}=\frac{b s i}{s+i}-d_{1} i-\frac{c(1-m) i y}{y+(1-m) i}, \quad i(0) \geq 0 \\
& \frac{d y}{d t}=y\left[\frac{l(1-m) i(t-\tau)}{y(t-\tau)+(1-m) i(t-\tau)}-d_{2}\right], \quad y(0) \geq 0
\end{aligned}
$$

All parameters are the same as in system (2.2) except that the positive constant $\tau$ represents the reaction time or gestation period of the predator $y$.

The system (6.1) has the same equilibria as in the previous case. The main purpose of this section is to study the stability behaviour of $E^{*}\left(s^{*}, i^{*}, y^{*}\right)$ in the presence of discrete delay $(\tau \neq 0)$. Before we get into the depth of the things, we would like to consider the following theorem, which gurantees the boundedness of the time-delayed system (6.1).

Theorem 6.1. If $l>d_{2}$, all the solutions of the system(6.1) that start in $\Re_{+}^{3}$ are bounded.

Proof : First observe that

$$
\limsup _{t \rightarrow \infty} s(t) \leq 1
$$

Proceeding as in the proof of theorem 3.1, we obtain

$$
\frac{d}{d t}(b s+a i) \leq 2 b-\alpha(b s+a i), \text { where } \alpha=\min \left\{1, d_{1}\right\}
$$

and for $t \rightarrow \infty, \quad 0 \leq b s+a i \leq \frac{2 b}{\alpha}$.

Therefore, it is possible to find two positive numbers $\kappa$ and $T$ such that $i(t)<\kappa$ for $t>T$. Since

$$
\begin{aligned}
\frac{d y}{d t} & \leq\left\{l(1-m)-d_{2}\right\} y(t)\left\{\frac{i(t-\tau)}{y(t-\tau)+(1-m) i(t-\tau)}\right\} \\
& \leq\left\{l(1-m)-d_{2}\right\} y(t)
\end{aligned}
$$

We have, for $t>\tau, \quad y(t) \leq y(t-\tau) e^{\left\{l(1-m)-d_{2}\right\} \tau}$, which is equivalent to $y(t-\tau) \geq y(t) e^{-\left\{l(1-m)-d_{2}\right\} \tau}$ 
Therefore, for $t>T+\tau$, we have

$$
\begin{aligned}
\frac{d y}{d t} & \leq y(t)\left\{\frac{l(1-m) \kappa}{y(t) e^{-\left\{l(1-m)-d_{2}\right\} \tau}+(1-m) \kappa}-d_{2}\right\} \\
& =y(t)\left\{\frac{(1-m) \kappa\left(l-d_{2}\right)-d_{2} y(t) e^{-\left\{l(1-m)-d_{2}\right\} \tau}}{y(t) e^{-\left\{l(1-m)-d_{2}\right\} \tau}+(1-m) \kappa}\right\}
\end{aligned}
$$

A Standard comparison argument shows that

$$
\limsup _{t \rightarrow \infty} y(t) \leq \frac{(1-m)\left(l-d_{2}\right) \kappa}{d_{2}} e^{\left\{l(1-m)-d_{2}\right\} \tau} .
$$

Hence the theorem.

We now study the stability behaviour of $E^{*}\left(s^{*}, i^{*}, y^{*}\right)$ for the system (6.1). We linearize system (6.1) by using the following transformation :

$$
s=s^{*}+s_{1}, \quad i=i^{*}+i_{1}, \quad y=y^{*}+y_{1}
$$

Then linear system is given by

$$
\frac{d U}{d t}=A U(t)+B U(t-\tau)
$$

where

$$
U(t)=\left[\begin{array}{lll}
s_{1} & i_{1} & y_{1}
\end{array}\right]^{T}, \quad A=\left(a_{i j}\right)_{3 \times 3}, \quad B=\left(b_{i j}\right)_{3 \times 3}
$$

and

$$
\begin{gathered}
a_{11}=-s^{*}+\frac{a s^{*} i^{*}}{\left(s^{*}+i^{*}\right)^{2}}, \quad a_{12}=-s^{*}-\frac{a s^{*^{2}}}{\left(s^{*}+i^{*}\right)^{2}}, \quad a_{21}=\frac{b i^{*^{2}}}{\left(s^{*}+i^{*}\right)^{2}}, \quad a_{22}=-\frac{b s^{*} i^{*}}{\left(s^{*}+i^{*}\right)^{2}}+\frac{c(1-m)^{2} i^{*} y^{*}}{\left\{y^{*}+(1-m) i^{*}\right\}^{2}} \\
a_{23}=-\frac{c(1-m)^{2} i^{*^{2}}}{\left\{y^{*}+(1-m) i^{*}\right\}^{2}}, \quad \text { and all other } a_{i j}=0, \quad b_{32}=\frac{l(1-m) y^{*^{2}}}{\left\{y^{*}+(1-m) i^{*}\right\}^{2}}, \quad b_{33}=-\frac{l(1-m) i^{*} y^{*}}{\left\{y^{*}+(1-m) i^{*}\right\}^{2}}
\end{gathered}
$$

and other $b_{i j}=0$.

We look for the solution of the model (6.2) of the form $U(t)=\rho e^{\lambda t}, \quad 0 \neq \rho \in \Re$. This leads to the following characteristic equation :

$$
\lambda^{3}+a_{1} \lambda^{2}+a_{2} \lambda+\left(a_{3} \lambda^{2}+a_{4} \lambda+a_{5}\right) e^{-\lambda \tau}=0
$$

where

$$
\begin{gathered}
a_{1}=-a_{11}-a_{22}, \quad a_{2}=a_{11} a_{22}-a_{12} a_{21}, \quad a_{3}=-b_{33}, \\
a_{4}=\left(a_{11}+a_{22}\right) b_{33}-a_{23} b_{32}, \quad a_{5}=a_{12} a_{21} b_{33}-a_{11}\left(a_{22} b_{33}-a_{23} b_{32}\right)
\end{gathered}
$$

It is well known that the signs of the real parts of the solutions of (6.3) characterize the stability behaviour of $E^{*}$. Therefore, substituting $\lambda=\xi+i \eta$ in (6.3) we obtain real and imaginary parts, respectively as

$$
\xi^{3}-3 \xi \eta^{2}+a_{1} \xi^{2}-a_{1} \eta^{2}+a_{2} \xi+e^{-\xi \tau}\left[\left\{a_{3}\left(\xi^{2}-\eta^{2}\right)+a_{4} \xi+a_{5}\right\} \cos \eta \tau+\left(2 a_{3} \xi+a_{4}\right) \eta \sin \eta \tau\right]=0
$$

and

$$
3 \xi^{2} \eta-\eta^{3}+2 a_{1} \xi \eta+a_{2} \eta+e^{-\xi \tau}\left[\left(2 a_{3} \xi+a_{4}\right) \eta \cos \eta \tau-\left\{a_{3}\left(\xi^{2}-\eta^{2}\right)+a_{4} \xi+a_{5}\right\} \sin \eta \tau\right]=0
$$

A necessary condition for a stability change of $E^{*}$ is that the characteristic equation (6.3) should have purely imaginary solutions. Hence to obtain the stability criterion, we set $\xi=0$ in (6.4) and (6.5). Then we have,

$$
\begin{gathered}
a_{1} \eta^{2}=\left(a_{5}-a_{3} \eta^{2}\right) \cos \eta \tau+a_{4} \eta \sin \eta \tau \\
-\eta^{3}+a_{2} \eta=\left(a_{5}-a_{3} \eta^{2}\right) \sin \eta \tau-a_{4} \eta \cos \eta \tau
\end{gathered}
$$

Eliminating $\tau$ by squaring and adding (6.6) and (6.7), we get the equation for determining $\eta$ as

$$
\eta^{6}+d_{1} \eta^{4}+d_{2} \eta^{2}+d_{3}=0
$$

where

$$
d_{1}=a_{1}^{2}-2 a_{2}-a_{3}^{2}, \quad d_{2}=a_{2}^{2}+2 a_{3} a_{5}-a_{4}^{2}, \quad d_{3}=-a_{5}^{2}
$$

Substituting $\eta^{2}=\sigma$ in (6.8), we get a cubic equation given by

$$
\sigma^{3}+d_{1} \sigma^{2}+d_{2} \sigma+d_{3}=0
$$


By Descartes' rule, the cubic (6.9) always have at least one positive root. Consequently the stability criteria of the system for $\tau=0$ (i.e. of the system 2.2) will not necessarily ensure the stability of the system for $\tau \neq 0$. In the following theorem, we have given a criterion for switching the stability behaviour of $E^{*}$.

Theorem 6.2. Let $E^{*}$ exists with $A_{1}>0, A_{3}>0$ \& $\Delta>0$. Also let $\sigma_{0}=\eta_{0}^{2}$ be a positive root of (6.9). Then there exists a $\tau=\tau^{*}$ such that $E^{*}$ is locally asymptotically stable for $0 \leq \tau<\tau^{*}$ and unstable for $\tau>\tau^{*}$, provided

$$
\begin{gathered}
f\left(\eta_{0}\right)=\left(2 a_{1}^{2} \eta_{0}^{2}-a_{4}^{2}\right)+2 a_{3}\left(a_{5}-a_{3} \eta_{0}^{2}\right)+\left(a_{2}-3 \eta_{0}^{2}\right)\left(a_{2}-\eta_{0}^{2}\right)>0 \\
\eta^{*}=\min g\left(\eta_{0}\right)
\end{gathered}
$$

where $g\left(\eta_{0}\right)=\frac{1}{\eta_{0}} \arccos \left[\frac{\eta_{0}^{2}\left\{a_{1}\left(a_{5}-a_{3} \eta_{0}^{2}\right)-a_{4}\left(a_{2}-\eta_{0}^{2}\right)\right\}}{\left(a_{5}-a_{3} \eta_{0}^{2}\right)^{2}-a_{4}^{2} \eta_{0}^{2}}\right]$ and the minimum is taken over all positive $\eta_{0}$ such that $\eta_{0}^{2}$ is a solution of the equation (6.9).

Or, in other words, the system (6.1) exhibits a hopf bifurcation near $E^{*}$ for $\tau=\tau^{*}$. (Expressions for $A_{1}, A_{3}, \Delta$ are given in section 5).

Proof : Notice that $\eta_{0}$ is a solution of (6.8). Solving (6.6) for $\cos \tau \eta_{0}$ and substituting in (6.7), we find that for $\tau=\tau_{0}$, the characteristic equation (6.3) has purely imaginary roots, $\pm i \eta_{0}$, where $\tau_{0}=g\left(\eta_{0}\right)$. Again it may be noted that if $\pm i \eta_{0}$ is a solution of (6.6) and (6.7), then $\eta_{0}^{2}$ is a solution of (6.8). Then smallest value of such a $\tau_{0}$ gives the required $\tau^{*}$. The theorem will be proved if we can show that $\left[\frac{d \xi}{d \tau}\right]_{\tau=\tau^{*}}>0$. To show this, we differentiate (6.6) and (6.7) with respect to $\tau$ and then set $\xi=0$ to obtain

$$
\begin{aligned}
& C(\eta) \frac{d \xi}{d \tau}+D(\eta) \frac{d \eta}{d \tau}=G(\eta) \\
& -D(\eta) \frac{d \xi}{d \tau}+C(\eta) \frac{d \eta}{d \tau}=H(\eta)
\end{aligned}
$$

where

$$
\begin{aligned}
& C(\eta)=-3 \eta^{2}+a_{2}+a_{4} \cos \eta \tau+2 a_{3} \eta \sin \eta \tau-\left(a_{5}-a_{3} \eta^{2}\right) \tau \cos \eta \tau-a_{4} \eta \tau \sin \eta \tau \\
& D(\eta)=-2 a_{1} \eta-2 a_{3} \eta \cos \eta \tau-\left(a_{5}-a_{3} \eta^{2}\right) \tau \sin \eta \tau+a_{4} \sin \eta \tau+a_{4} \eta \tau \cos \eta \tau \\
& G(\eta)=\left(a_{5}-a_{3} \eta^{2}\right) \eta \sin \eta \tau-a_{4} \eta^{2} \cos \eta \tau \\
& H(\eta)=a_{4} \eta^{2} \sin \eta \tau+\left(a_{5}-a_{3} \eta^{2}\right) \eta \cos \eta \tau
\end{aligned}
$$

Solving (6.11) and (6.12) with $\tau=\tau^{*}$ and $\eta=\eta_{0}$, we get

$$
\left[\frac{d \xi}{d \tau}\right]_{\tau=\tau^{*}}=\frac{\eta_{0}^{2} f\left(\eta_{0}\right)}{C\left(\eta_{0}\right)^{2}+D\left(\eta_{0}\right)^{2}}
$$

which is positive under the condition (6.10), and thus the theorem established.

\section{Numerical Simulation}

Analytical studies can never be completed without numerical verification of the results. In this section we present computer simulation of some solutions of the system (2.2) and (6.1). Beside verification of our analytical findings, these numerical solutions are very important from practical point of view.

We choose the parameters of system $(2.2)$ as $a=1.2, b=0.75, d_{1}=0.2, c=1.0, d_{2}=0.8, l=2.0$ and $(s(0), i(0), y(0))=(0.5,0.5,0.5)$. Also we choose $m=0.6$ which satify the inequality (5.1). Then the conditions of Theorem 5.1 is satisfied as $A_{1}=0.5706>0, \Delta=0.0461>0$ and consequently $E^{*}\left(s^{*}, i^{*}, y^{*}\right)=$ $(0.2957,0.2089,0.1250)$ is locally asymptotically stable. The phase diagram is shown in Fig. 1a. The si-plane and iy-plane projections of the solution are shown in Fig. 1b and 1c respectively. Clearly the solution is a stable spiral converging to $E^{*}$. Fig. 1d shows that s,i and y populations approach their steady-state values $s^{*}$, $i^{*}$, and $y^{*}$ respectively, in finite time. 


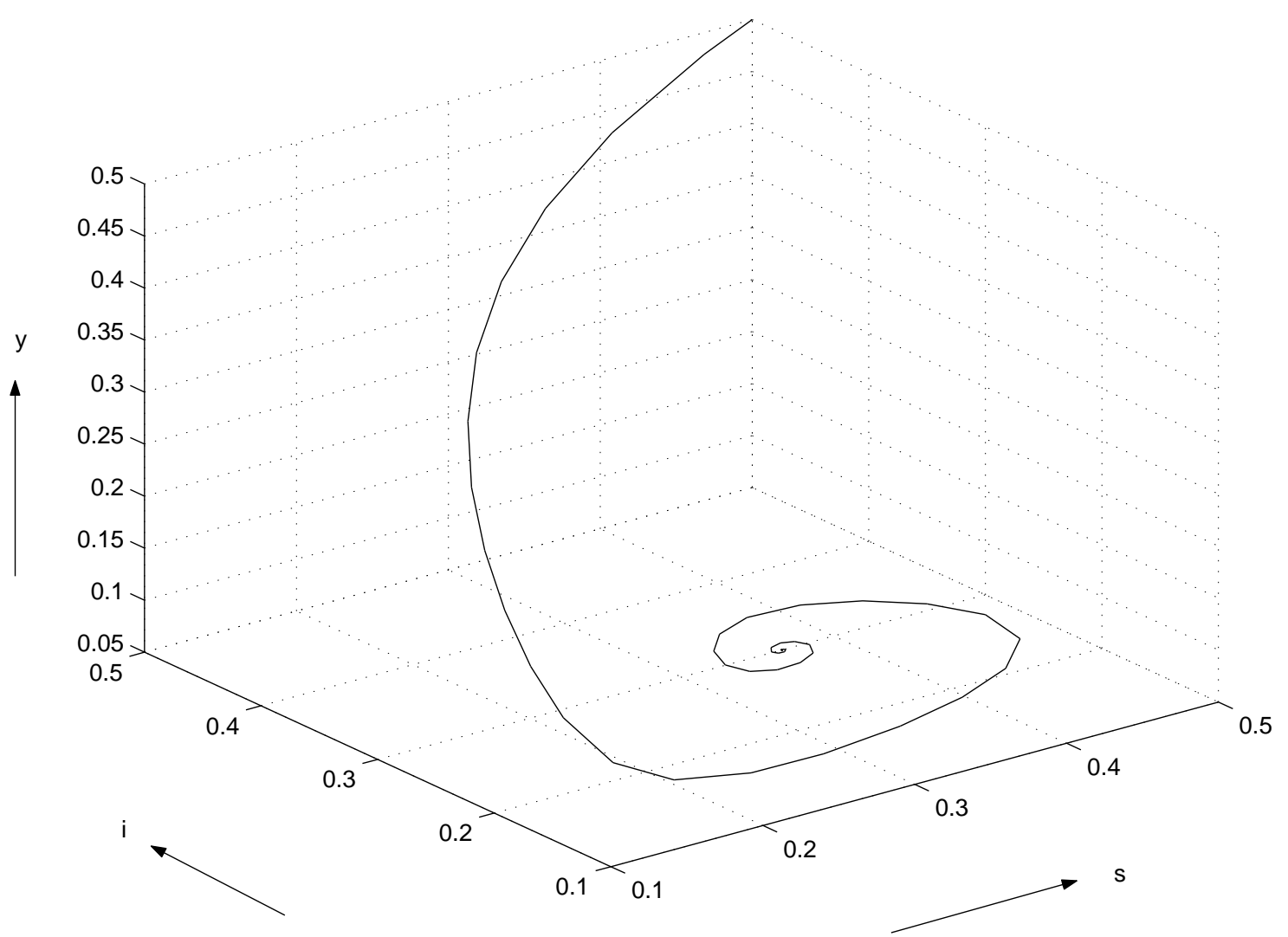

Fig.1a

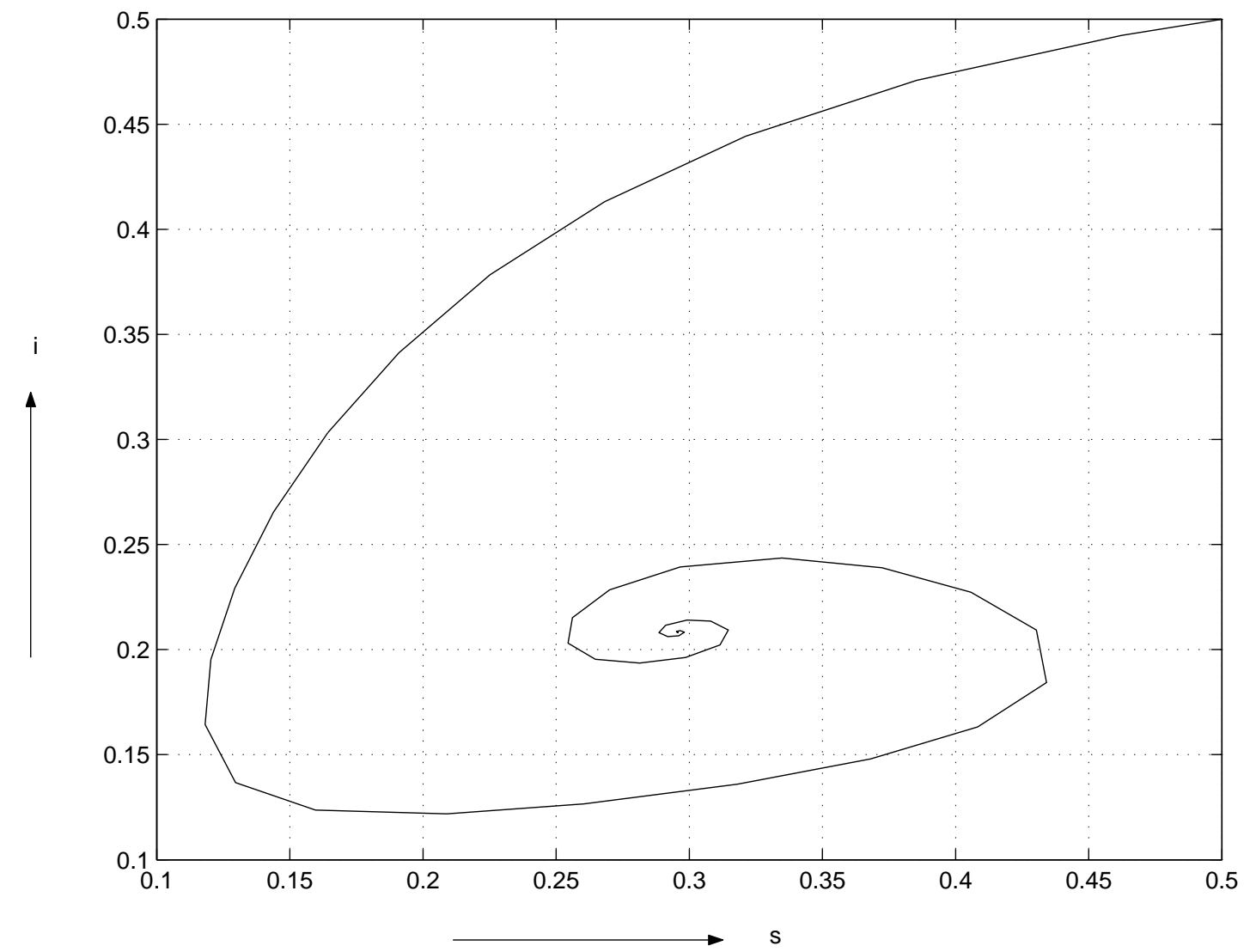

Fig.1b 


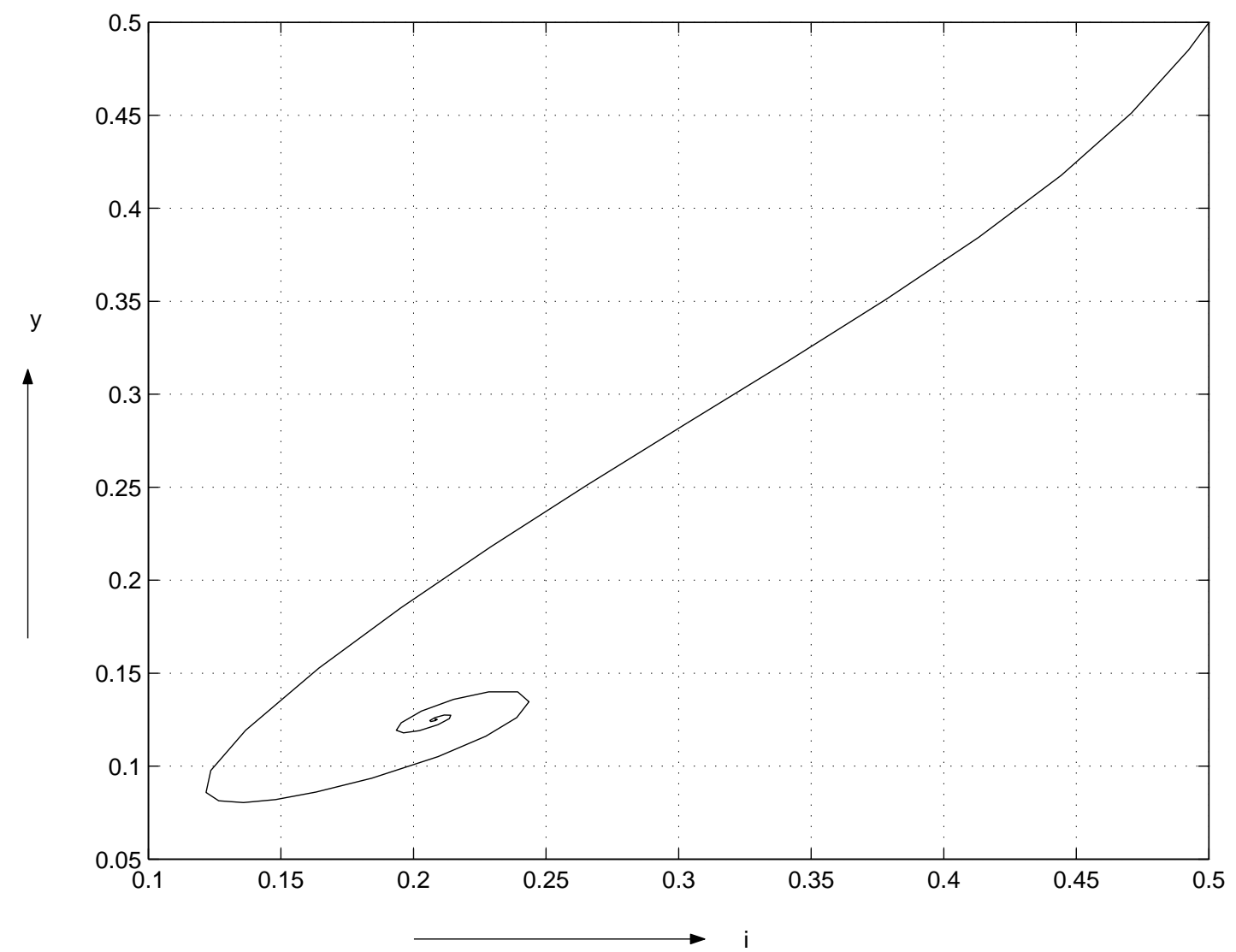

Fig.1c

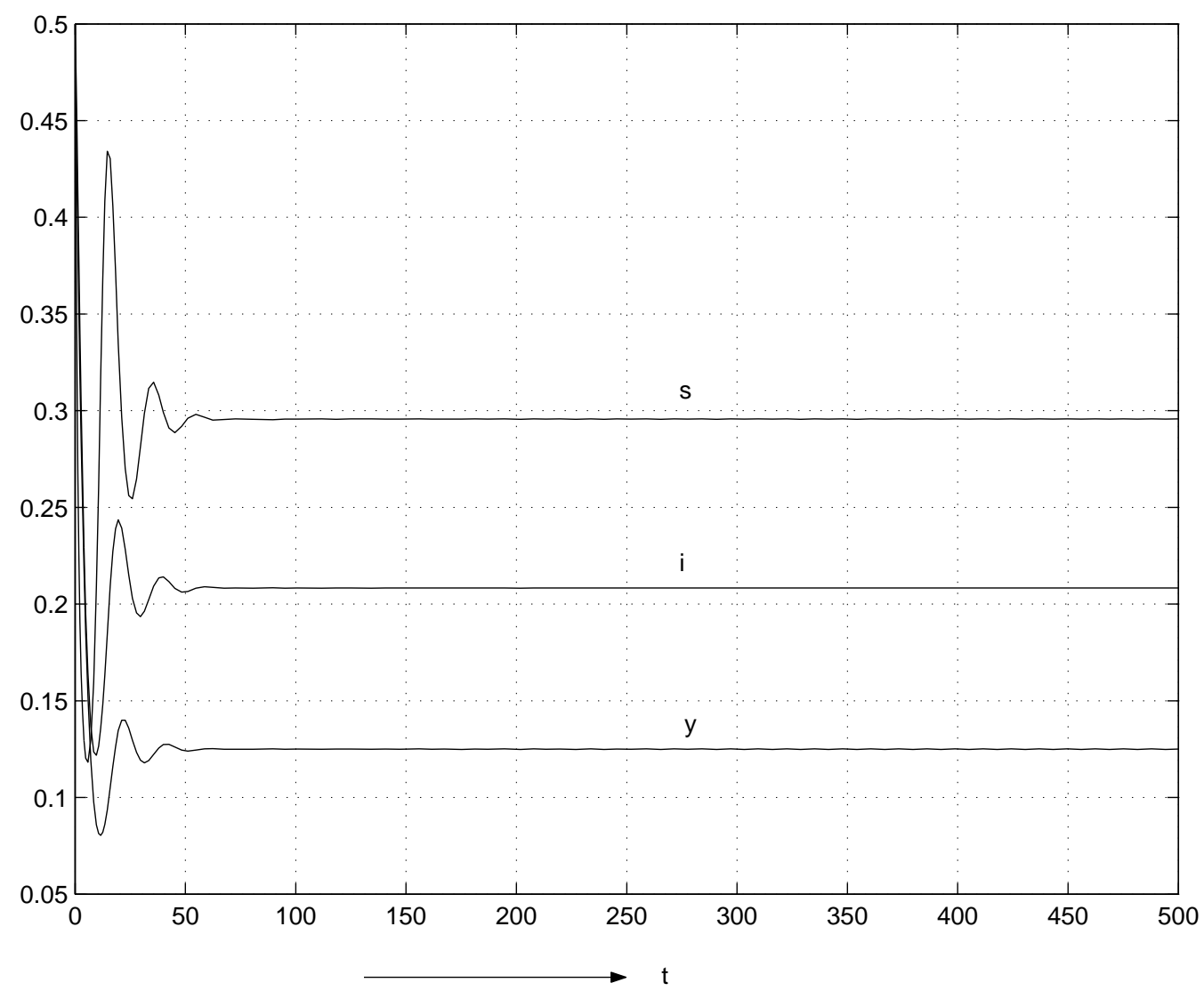

Fig.1d

Fig. 1. Here $s(0)=0.5, i(0)=0.5, y(0)=0.5$ and $a=1.2, b=0.75, d_{1}=0.2, c=1.0, d_{2}=0.8, l=$ 2.0, $m=0.6$. (a) Phase portrait of the system (2.2) showing that $E^{*}$ is locally asymptotically stable. (b) si-plane projection of the solution. (c) iy-plane projection of the solution. (d) Top curve depicts $s(t)$, middle one depicts $i(t)$ and the bottom one depicts $y(t)$. Clearly the $s, i, y$ populations approach to their equilibrium values in finite time. 
It is mentioned before that the stability criteria in the absence of delay $(\tau=0)$ will not necessarily guarantee the stability of the system in presence of delay $(\tau \neq 0)$. Let us choose the parameters of the system as $a=1.2, b=$ $0.75, d_{1}=0.2, c=1.0, d_{2}=0.8, l=2.0, m=0.6$ and $(x(0), y(0), z(0))=(0.5,0.5,0.5)$. It is already seen that for such choices of parameters $E^{*}=(0.2957,0.2083,0.1250)$ is locally asymptotically stable in the absence of delay. Now for these choices of parameters, it is seen from Theorem 6.2 that there is a unique positive root of (6.9) given by $\sigma_{0}=\eta_{0}^{2}=0.2526$ for which $f\left(\eta_{0}\right)=0.0330>0$ and $\tau=\tau^{*}=2.7379$. Therefore by Theorem 7.2 , $E^{*}=\left(s^{*}, i^{*}, y^{*}\right)$ losses its stability as $\tau$ passes through the critical value $\tau^{*}$. We verify that for $\tau=2.47<\tau^{*}, E^{*}$ is locally asymptotically stable, the phase portrait of the solution (presented in Fig. 2a) being stable spiral. Fig. $2 \mathrm{~b}$ shows that for the above choices of parameters, s, i, y populations converge to their equilibrium values $s^{*}, i^{*}$, $y^{*}$, respectively. Keeping other parameters fixed, if we take $\tau=3.0>\tau^{*}$, it is seen that $E^{*}$ is unstable and there is a bifurcating periodic solution near $E^{*}$ (see Fig. 3a). Fig. 3b, 3c, 3d depicts the oscillations of the populations $\mathrm{s}, \mathrm{i}, \mathrm{y}$ in finite time.

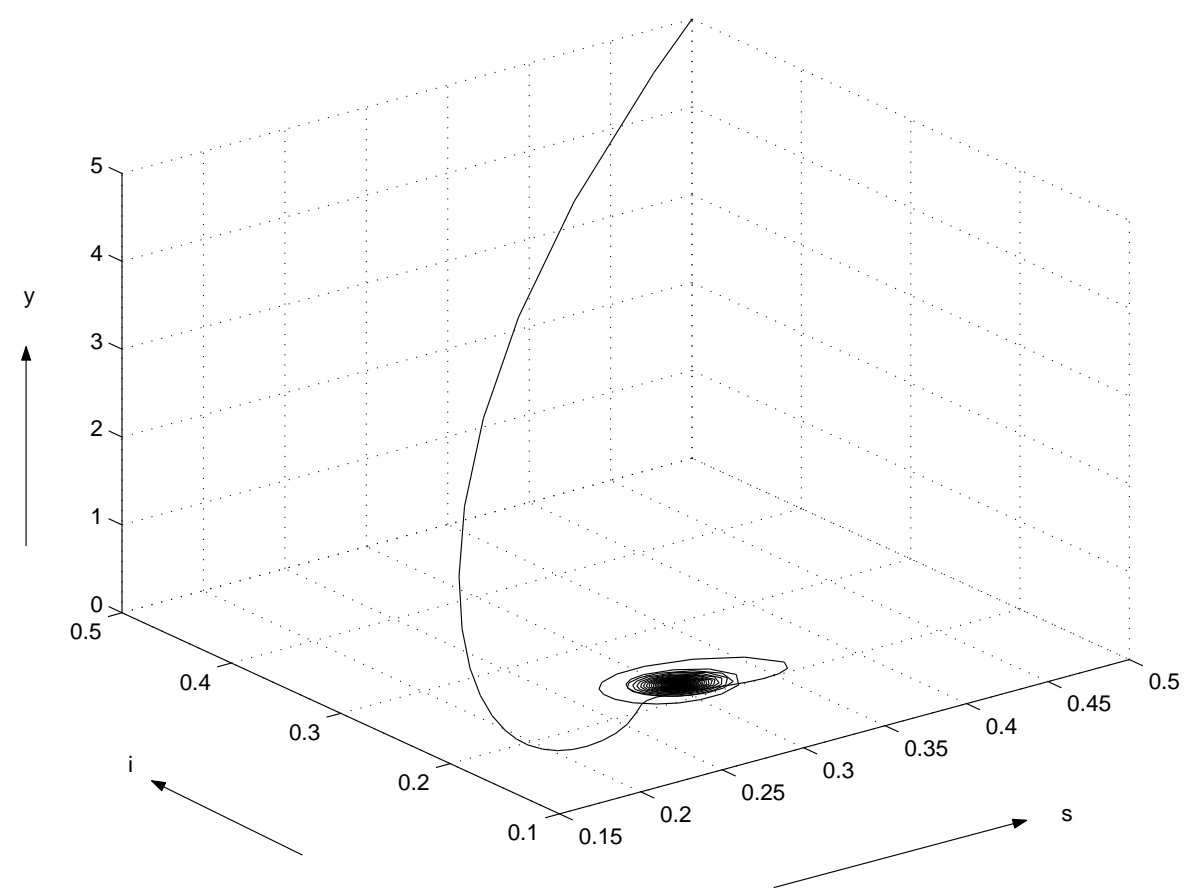

Fig.2a

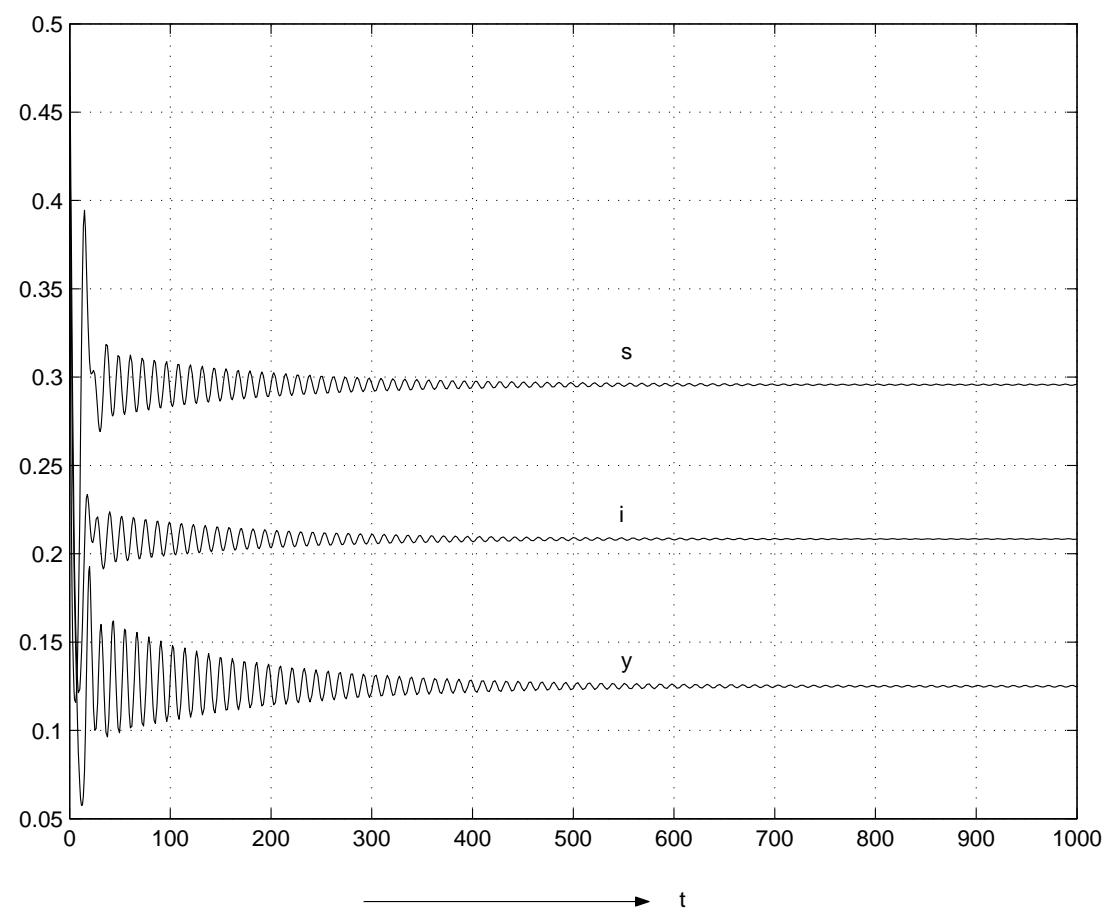

Fig.2b

Fig. 2. Here $x(0)=0.5, y(0)=0.5, z(0)=0.5$ and $a=1.2, b=0.75, d_{1}=0.2, c=1.0, d_{2}=0.8, l=2.0, m=$ 0.6 , and $\tau=2.47<\tau^{*}$. (a) Phase portrait of the system. (b) Stable behaviour of $s$, $i, y$ in time. 


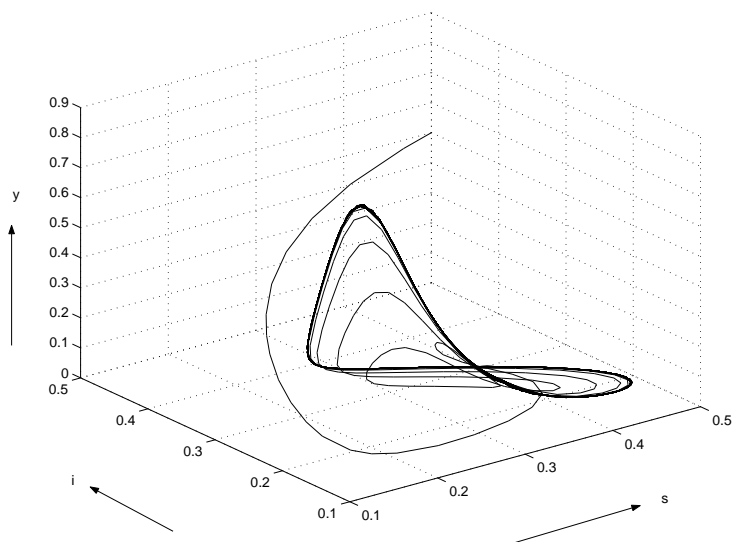

Fig.3a

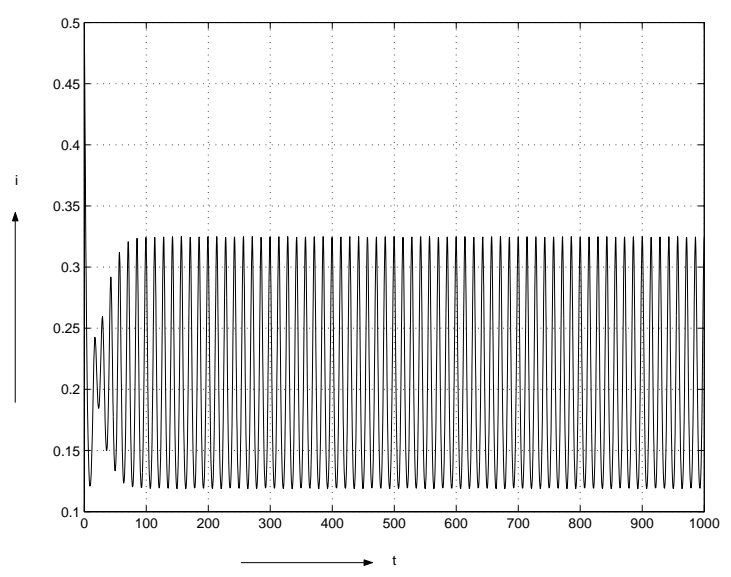

Fig.3c

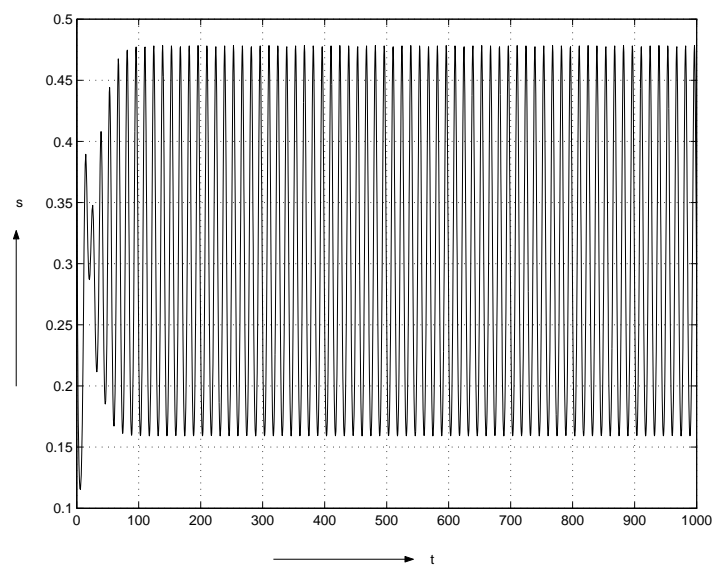

Fig.3b

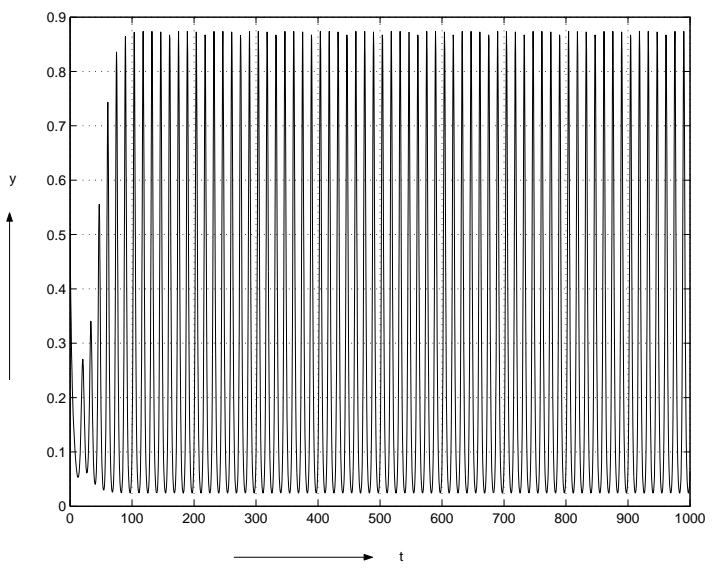

Fig.3d

Fig. 3. Here all other parameter values are same as in Fig. 2 except $\tau=3.0>\tau^{*}$. (a) Phase portrait of the system (6.1) showing a limit cycle which grows out of $E^{*} .(b),(c)$ and (d) Oscillations of s, $i$ and $y$ respectively in time.

\section{Discussion}

In this paper, we have studied an eco-epidemiological model incorporating an prey refuge with disease in the prey population which is governed by modified logistic equation [32]. Incorporating a refuge into system (2.1) provides a more realistic model. A refuge can be important for the biological control of a pest, however, increasing the amount of refuge can increase prey densities and lead to population outbreaks. It is shown (in Theorem 3.1) that the non-dimensionalized system (2.2) is uniformly bounded, which in turn, implies that the system is biologically well behaved. In deterministic situation, theoretical epidemiologists are usually guided by an implicit assumption that most epidemic models we observe in nature correspond to stable equilibria of the models. From this viewpoint, we have presented the most important equilibrium point $E^{*}\left(s^{*}, i^{*}, y^{*}\right)$. The stability criteria given in Lemma 5.1 and Theorem 5.1 are the conditions for stable coexistence of the susceptible prey population, infected prey population and predator population.

Now we observe that the size of the susceptible prey $(s)$ in the absence and presence of infected prey $(i)$ are $\hat{s}=$ $\frac{d_{1}}{b^{2}}\left\{b-a\left(b-d_{1}\right)\right\}$ and $s^{*}=\frac{A}{l^{2} b^{2}}\{l b-a(l b-A)\}$, respectively, so that $s^{*}-\hat{s}=\frac{c(1-m)\left(l-d_{2}\right)}{l^{2} b^{2}}\left[\{l b-a(l b-A)\}+a l d_{1}\right]>$ 0 whenever $l>d_{2}$. This indicates that if the force of infection is high then predator attack causes enhancement of the susceptible preys.

It is mentioned by several researchers that the effect of time-delay must be taken into account to have a epidemiologically useful mathematical model [8,10,21,27]. From this viewpoint, we have formulated (6.1) where the delay may be looked upon as the gestation period or reaction time of the predator. Then a rigorous analysis leads us to Theorem 7.2 which mentions that the stability criteria in absence of delay is no longer enough to guarantee the stability in the presence of delay, rather there is a value $\tau^{*}$ of the delay $\tau$ such that the system is stable for $\tau<\tau^{*}$ and become unstable for $\tau>\tau^{*}$. 
All our important mathematical findings without and with time-delay are numerically verified and graphical representation of a variety of solutions of system (2.2) and (6.1) are depicted using MATLAB. Our analytical and numerical studies show that, using the delay $\tau$ as control, it is possible to break the stable (spiral) behaviour of the system and drive it to an unstable (cyclic) state. Also it is possible to keep the levels of prey population (susceptible and infectious) and predator at a required state using the above control.

Finally, our model can be generalized in obvious ways to food chains and competitive systems.

\section{Acknowledgements}

The authors are grateful to the anonymous referees and Editor-in-Chief for their careful reading, valuable comments and helpful suggestions, which have helped us to improve the presentation of this work significantly.

\section{REFERENCES}

[1] R. M. Anderson and R. M. May, Infectious Disease of Human dynamics and Control, Oxford University Press, Oxford, 1991.

[2] N. J. T. Bailey, The Mathematical Theory of Infectious Disease and its Application, Griffin, London, 1975.

[3] E. Beretta and Y. Kuang, Global anlysis in some delayed retio-dependent predator-prey system, Nonlinear Analysis TMA, 32 (1998), 381-408.

[4] G. Birkhoff and G. C. Rota, Ordinary Differentiak Equations, Ginn, Boston, 1982.

[5] J. Chattopadhyay and O. Arino, A predator-prey model with disease in prey, Nonlinear Anal., 36 (1999), 747-766.

[6] L. H. Erbe, V. S. H. Rao and H. Freedman, Three-species food chain models with mutual interference and time delays, Math. Biosci., 80 (1986), 57-80.

[7] H. I. Freedman, Deterministic mathematical models in population ecology, Marcel dekker, New York, 1980.

[8] H. Freedman and V.S. H. Rao, The trade-off between mutual interference and tome lags in predator-prey systems, Bull. Math. Biol., 45 (1983), 991-1004.

[9] T. C. Gard and T. G. Hallam, Persistence in food web-1, Lotka-Volterra food chains, Bull. Math. Biol., 41 (1979), 302-315.

[10] K. Gopalsamy, Stability and Oscillation in Delay-Differential Equations of Population Dynamics, Kluwer, Dordrecht, 1992.

[11] K. P. Hadeler and H. I. Freedman, Predator-prey populations with parasite infection, J. Math. Biol., 27 (1989), 609-631.

[12] M. P. Hassel, The Dynamics of arthropod predator-prey Systems, Princeton, Princeton University Press, 1974.

[13] H. W. Hethcote, M. A. Lewis and P. van den Driessche, An epidemiological model with delay and a nonlinear incidence rate, J. Math. Biol.27 (1989), 49-64.

[14] H. W. Hethcote, W. Wang, L. Han and Z. Ma, A predator-prey model with infected prey, Theor. Pop. Biol., 34 (2004), 849-858.

[15] C. S. Holling, The componentsof predation as revealed by a study of small mammal predation of the European pine sawfly, Can. Entomol., 91 (1959), 293-320.

[16] Y. Huang, F. Chen and L. Zhong, Stability analysis of a prey-predator model with Holling type III response function incorporating a prey refuge, Appl. Math. Comp., 182 (2006), 672-683.

[17] P. J. Hudson, A. P. Dobson and D. Newborn, Do parasite make prey vulnerable to predation Red grouse and parasite, J. Animal Ecol., 61 (1992), 681-692.

[18] T. W. Hwang and Y. Kuang, Deterministic extinction effects of parasites on host population, J. Math. Biol., 46 (2003), $17-30$.

[19] T. K. Kar, Stability analysis of a prey-predator model incorporating a prey refuge, Comm. Nonlinear Sc. and Numerical Simul.,10 (2005), 681-691.

[20] W. O. Kermack and A. G. McKendrick, Contributions to the mathematical theory of epidemics, part 1, Proc. R. Soc. London, A 115, (1927), 700-721. 
[21] Y. Kuang, Delay Differential Equations with Applications in Population Dynamics, Academic Press, New York, 1993.

[22] W. Liu, Criterion of Hopf bifurcations without using eigenvalues, J. Math. Anal. Appl., 182 (1994), $250-256$.

[23] W. M. Liu, H. W. Hethcote and S. A. Levin, Influence of nonlinear incidece rates upon the behaviour of SIRS epidemiological models, J. Math. Biol., 23 (1986), 187-204.

[24] W. M. Liu, S. A. Levin and Y. Iwasa, Dynamical behaviour of epidemiological models with nonlinear incidence rates, J. Math. Biol., 25 (1987), 359-380.

[25] A. J. Lotka, Elements of Physical Biology, Williams and Wilkins Co., Inc., Baltimore, 1924.

[26] Z. Ma, W. Li, Y. Zhao, W. Wang, H. Zhang, and Z. Li Effects of prey refuges on a predator-prey model with a class of functional responses: the role of refuges, Mathematical Biosciences, 218(2)(2009), 73-79.

[27] N. Macdonald, Time delays in Biological Models, Spring, Heidelberg, 1978.

[28] J. D. Murray, Mathematical Biology, Spinger-Verlag, Newyork, 1993.

[29] V. Volterra, Variazioni e fluttauazionidel numero d individui in specie animals conviventi, Mem. Acad. Lincei, 2, (1926), 31-33.

[30] S. Wang and Z. Ma, Analysis of an Ecoepidemiological Model with Prey Refuges, Journal of Applied Mathematics Volume 2012, Article ID 371685, 16 pages, doi:10.1155/2012/371685.

[31] D. Xiao and S. Ruan, Global dynamics of a ratio-dependent predator-prey system, J. Math. Biol., 43(2001), 268-290.

[32] Y. Xiao and L. Chen, A ratio-dependent Predator-prey model with disease in the prey, Appl. Math. Comp., 131 (2002), 397-414. 